\title{
Book Review · Buchbesprechung · Livre nouveau
}

H.M. Van Praag (ed.): On the Origin of Schizophrenic Psychoses. De Erven Bohn, Amsterdam, 1975. VIII + 222 pp.; Fl. 45.-; paperback.

This book includes various studies presented during two recent meetings of the Interdisciplinary Society of Biological Psychiatry. There are 15 chapters dealing with different etiological aspects of schizophrenic psychoses. Most chapters are based on original research work carried out by distinguished investigators in the field of schizophrenic research.

The biological factors are stressed by genetic, biochemical and neurophysiological studies, but there are also some interesting etiological, social and psychodynamic analyses. Among those are excellent studies such as the one described by A.R. Cools on 'An integrated theory of the etiology of schizophrenia', where the author describes neurophysiological theories in relation with dopaminergic, noradrenergic and serotonergic mechanisms in the etiology of schizophrenia. The work presented by S.J. Hutt is also fascinating in establishing correlations between autistic behavior and neurophysiological variables. The genetic approach, although very promising, especially for the adoption studies described by $S$. Kety, is a bit summarized and leaves the reader somewhat frustrated. In any event, this is an excellent book presenting a large spectrum on recent research in the etiology of schizophrenia and it has the quality of including biological, psychological as well as social factors.

J.M. 